\title{
Global use of Haemophilus influenzae type b conjugate vaccine ${ }^{\text {is }}$
}

\author{
Linda R. Ojo a, Rosalyn E. O'Loughlin ${ }^{\mathrm{b}}$, Adam L. Cohen ${ }^{\mathrm{a}, *}$, Jennifer D. Loo ${ }^{\mathrm{a}}$, Karen M. Edmond ${ }^{\mathrm{b}}$, \\ Sharmila S. Shetty ${ }^{c}$, Allyson P. Bear ${ }^{c}$, Lois Privor-Dumm ${ }^{c}$, Ulla K. Griffiths ${ }^{b}$, Rana Hajjeh ${ }^{\mathrm{a}}$ \\ a Respiratory Diseases Branch, Division of Bacterial Diseases, Centers for Disease Control and Prevention, 1600 Clifton Road NE, MS C-23, Atlanta, GA 30333, USA \\ ${ }^{\mathrm{b}}$ Department of Epidemiology and Population Health, London School of Hygiene and Tropical Medicine, London, United Kingdom \\ ${ }^{\mathrm{c}}$ Department of International Health, Johns Hopkins Bloomberg School of Public Health, Baltimore, USA
}

\section{A R T I C L E I N F O}

\section{Article history:}

Received 26 March 2010

Received in revised form 20 July 2010

Accepted 21 July 2010

Available online 4 August 2010

\section{Keywords:}

Haemophilus influenzae type b

Vaccine

Developing countries

\begin{abstract}
A B S T R A C T
Haemophilus influenzae type b (Hib) conjugate vaccines have been underutilized globally. We report progress in global use of Hib vaccines included in national immunization schedules. The number of countries using Hib vaccine increased from 89/193 (46\%) in 2004 to 158/193 (82\%) by the end of 2009. The increase was greatest among low-income countries eligible for financial support from the GAVI Alliance [ $13 / 75$ (17\%) in 2004, 60/72 (83\%) by the end of 2009], and can be attributed to various factors. Additional efforts are still needed to increase vaccine adoption in lower middle income countries [20/31 (65\%) by the end of 2009].
\end{abstract}

Published by Elsevier Ltd.

\section{Introduction}

Haemophilus influenzae type $\mathrm{b}$ (Hib) disease is a common cause of morbidity and mortality in children under 5 years of age in developing countries. Globally, an estimated 8 million cases of pneumonia and meningitis and 371,000 deaths per year are attributed to Hib [1]. Pneumonia is the leading cause of child mortality globally, causing approximately $18 \%$ of deaths in children $<5$ years of age [2], and Hib disease has been estimated to account for $5 \%$ of clinical pneumonia cases and $21 \%$ of all radiologically defined pneumonia cases [1].

Hib conjugate vaccine has the potential to reduce overall childhood deaths by $4 \%$ [3], and incorporation into countries' routine immunization schedules has been recognized as an important indicator [4] of progress towards the fourth United Nations Millennium Development Goal of a two-thirds reduction in childhood mortality by 2015 [5]. Safe and effective Hib conjugate vaccines have been available since the late 1980s, but they have historically been underutilized, particularly in lower income countries. A field efficacy trial in The Gambia demonstrated a vaccine efficacy of $95 \%$ against invasive Hib disease [6], and where introduced, routine use of the vaccine has led to the virtual elimination of Hib disease [7-9].

\footnotetext{
The authors alone are responsible for the views expressed in this publication and they do not necessarily represent the decisions, policy or views of their respective organizations.

* Corresponding author. Tel.: +1 404639 6417; fax: +1 4046393970.

E-mail address: ALCohen1@cdc.gov (A.L. Cohen).
}

The GAVI Alliance (formerly known as the Global Alliance for Vaccines and Immunization) is a public-private partnership formed in 1999 with the goal of accelerating the uptake and use of new and underutilized vaccines in the poorest countries of the world. Between 2000 and 2004, uptake of Hib vaccine in GAVIeligible countries was slow despite free provision of the vaccine through the GAVI Alliance. The GAVI Alliance created the Hib Initiative in 2005 to accelerate evidence-based decision making for Hib vaccine introduction in GAVI-eligible countries. In this paper, we describe the progress made in the global use of Hib vaccine by income status and geographic location of the World Health Organization (WHO) members states, including both GAVI-eligible and ineligible countries.

\section{Materials and methods}

We identified WHO member states that use Hib conjugate vaccine in their infant immunization schedule through official 2008 WHO/United Nations Children's Fund (UNICEF) reports that provide comprehensive, country-level data on vaccines included in routine immunization systems, year of vaccine introduction, and vaccine coverage [10]. We supplemented these data with information collected from WHO; the GAVI Alliance (www.gavialliance.org); the Hib Initiative (www.hibaction.org); vaccine information management system (VIMS), a web-based vaccine information database based at Johns Hopkins University [11]; vaccine manufacturers; and direct contact with country officials. We considered a country to have introduced Hib conjugate vaccine if they used any of the more than 30 existing products and 


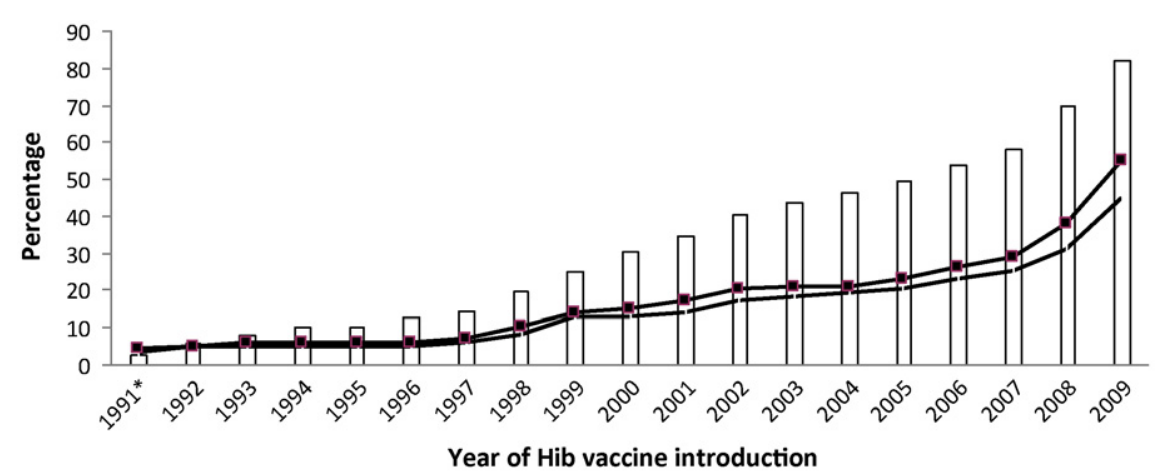

\begin{tabular}{|l|}
$\square \%$ of countries that introduced Hib vaccine \\
$-\%$ of birth cohort that received Hib vaccinet \\
- \% of birth cohort with access to Hib vaccine $\neq$
\end{tabular}

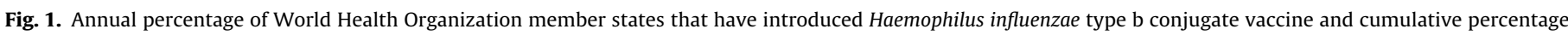

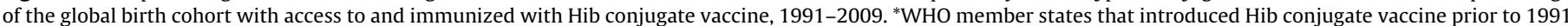

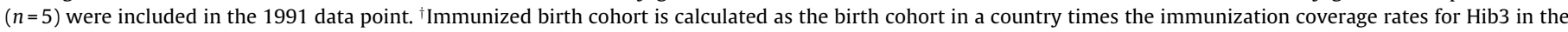
country. ${ }^{\ddagger}$ Access is considered to be the full birth cohort in a country that introduced the vaccine.

formulations that contain Hib conjugate vaccine in their national infant immunization schedule covering the entire country. Projections for after 2009 were based on countries that have been approved (including 1 country with conditional approval ${ }^{1}$ ) by the GAVI Alliance to introduce Hib vaccine, and for non-GAVI-eligible countries, on their expressed intention to WHO to introduce the vaccine. All data are based on information obtained as of December 2009.

We assessed two parameters to fully describe the global use of Hib vaccine. The first parameter is the number of infants with access to Hib vaccine approximated by the birth cohort of countries that have introduced the vaccine. We used 1991-2007 birth cohort data, with projections for 2008 and 2009, from the United Nations World Population Prospects database [12]. This was supplemented by data from WHO for 14 small countries not included in the World Population Prospects database [10]. The second parameter is the estimated number of infants fully vaccinated with Hib vaccine which we calculated by multiplying yearly UNICEF/WHO immunization coverage estimates for the third dose of Hib vaccine (Hib3) [10] by the respective annual birth cohort for each country that used the vaccine. Where data were not available for Hib3 coverage ( $n=23$ countries), mainly due to recent introduction, we used coverage rates for the 2008 third dose of diphtheria-tetanuspertussis (DTP3) as a proxy since both vaccines are administered on the same immunization schedule, and in many countries, as a combined preparation that contains both DTP and Hib vaccines.

To examine the income status of WHO member states that have introduced Hib vaccine into the routine infant immunization schedule, we used World Bank income indicators from 2003 [13] and GAVI Alliance income indicators from 2003 (the most recent year used for classification) to classify countries into four income strata: (1) 72 low income countries (GAVI-eligible), Gross National Income (GNI) per capita $\leq$ US\$ 1000; (2) 31 lower middle income countries, GNI US\$ 1001-US\$ 3705; (3) 38 upper middle income countries, GNI US\$3706-US\$ 11,455; and (4) 48 high income countries, GNI $\geq$ US\$ 11,456 . Four countries without GNI information (Cook Islands, Nauru, Niue, and Tuvalu) were excluded from this part of the analysis. To examine the geographical distribution of uptake of Hib vaccine, we categorized WHO member states based on the WHO regions: American, African, Eastern Mediterranean, European, South East Asian, and Western Pacific.

\footnotetext{
1 Countries with conditional approval are required to revise their application for re-submission to GAVI.
}

\section{Results}

Globally, the number of WHO member states using Hib conjugate vaccine has been slowly increasing since 1991 (Fig. 1). In the past 5 years, the number of WHO member states using Hib vaccine increased by $78 \%$ from $89 / 193$ (46\%) in 2004 to $158 / 193$ (82\%) in 2009 (Table 1). These 158 states are home to $55 \%$ of the world's infants, those who have access to Hib vaccine. By the end of 2009 , $45 \%$ of the world's infants are estimated to receive three doses of Hib vaccine based on WHO-UNICEF estimates of vaccine coverage. The number of WHO member states using Hib vaccine is projected to rise to $168 / 193$ (87\%) by the end of 2010 .

The greatest increase in the number of WHO member states using Hib vaccine occurred in GAVI-eligible (low income) countries, which increased from $13 / 75$ (17\%) in 2004 to $60 / 72(83 \%)$ by the end of 2009. It is estimated that as of December 2009, 49\% of infants in GAVI-eligible countries had access to and $38 \%$ were immunized with Hib vaccine. Based on available information from GAVI, the number of GAVI-eligible countries using Hib vaccine is projected to increase to 64/72 (89\%) countries by the end of 2010 . This increase represents three countries (Cambodia, Georgia and Vietnam) currently approved to introduce Hib vaccine by the GAVI Alliance. It also includes one country with conditional approval, Azerbaijan. Of the remaining eight GAVI-eligible countries, India has been approved and is still evaluating Hib vaccine introduction, Nigeria and Indonesia have applied for introduction but are not yet approved, and five countries (Haiti, Democratic People's Republic of Korea, Myanmar, Somalia, and Timor-Leste) have not yet applied to GAVI for vaccine financing. However, of these five countries, only one has not made a decision to introduce Hib vaccine into its national immunization program.

Higher income countries started introduction of Hib vaccine earlier than countries in lower income strata and have nearly achieved $100 \%$ Hib vaccine use (Fig. 2). Since 2000, all income strata have shown a steady increase in Hib vaccine uptake, with high income countries stabilizing in 2007 as only a few countries in this income classification have yet to introduce Hib vaccine. Between December 2007 and December 2009, a sharp increase in low income countries using Hib vaccine was observed, and is projected to continue through the end of 2010 due to the recent acceleration in decisions on introduction of Hib vaccine in GAVI-eligible countries. Of the 46 countries that introduced Hib vaccine in this period, 41 of those countries were GAVI-eligible countries. For the first time, in 2008, the proportion of GAVI-eligible countries using Hib vaccine was greater than the proportion of lower middle income countries 
Table 1

Global and GAVI-eligible Hib vaccine introduction status, 2004 and 2009.

\begin{tabular}{|c|c|c|c|c|c|c|}
\hline \multirow[t]{2}{*}{ Characteristics } & \multicolumn{3}{|c|}{ WHO member states } & \multicolumn{3}{|c|}{ GAVI-eligible countries } \\
\hline & No. & $\begin{array}{l}2004(n=193) \\
n(\%)\end{array}$ & $\begin{array}{l}2009(n=193) \\
n(\%)\end{array}$ & No. of countries & $\begin{array}{l}2004^{\mathrm{a}}(n=75) \\
n(\%)\end{array}$ & $\begin{array}{l}2009(n=72) \\
n(\%)\end{array}$ \\
\hline Countries using Hib vaccine & 193 & $89(46)$ & $158(82)$ & & $13(17)$ & $60(83)$ \\
\hline \multicolumn{7}{|c|}{ Countries using Hib vaccine, by income status ${ }^{\mathrm{b}}$} \\
\hline GAVI-eligible & 72 & $13(17)$ & $60(83)$ & 72 & $13(17)$ & $60(83)$ \\
\hline Lower middle income & 31 & $10(32)$ & $20(65)$ & $\mathrm{n} / \mathrm{a}$ & $\mathrm{n} / \mathrm{a}$ & $\mathrm{n} / \mathrm{a}$ \\
\hline Upper middle income & 38 & $22(58)$ & $33(87)$ & $\mathrm{n} / \mathrm{a}$ & $\mathrm{n} / \mathrm{a}$ & $\mathrm{n} / \mathrm{a}$ \\
\hline High income & 48 & $43(90)$ & $44(92)$ & $\mathrm{n} / \mathrm{a}$ & $\mathrm{n} / \mathrm{a}$ & $\mathrm{n} / \mathrm{a}$ \\
\hline \multicolumn{7}{|c|}{ Countries using Hib vaccine, by WHO region } \\
\hline American & 35 & $30(86)$ & $34(97)$ & 6 & $5(83)$ & $5(83)$ \\
\hline African & 46 & $9(20)$ & $41(89)$ & 36 & $8(22)$ & $35(97)$ \\
\hline Eastern Mediterranean & 21 & $10(48)$ & $16(76)$ & 6 & $0(0)$ & $5(83)$ \\
\hline European & 53 & $31(58)$ & $47(89)$ & 8 & $0(0)$ & $6(75)$ \\
\hline South East Asian & 11 & $0(0)$ & $4(36)$ & 9 & $0(0)$ & $4(44)$ \\
\hline Western Pacific & 27 & $9(33)$ & $16(59)$ & 7 & $0(0)$ & $5(71)$ \\
\hline \multicolumn{7}{|l|}{ Hib vaccine access and coverage } \\
\hline Birth cohort (combined) & $\mathrm{n} / \mathrm{a}$ & $134,515,686$ & $122,226,551$ & $\mathrm{n} / \mathrm{a}$ & $78,416,142$ & $80,855,633$ \\
\hline Children with access to Hib vaccine & $\mathrm{n} / \mathrm{a}$ & $28,627,232(21)$ & $67,066,722(55)$ & $\mathrm{n} / \mathrm{a}$ & $5,945,369(8)$ & $39,347,079(49)$ \\
\hline Children immunized with Hib3 & $\mathrm{n} / \mathrm{a}$ & $25,872,781$ (19) & $55,513,026(45)$ & $\mathrm{n} / \mathrm{a}$ & $4,867,903(6)$ & $30,360,872(38)$ \\
\hline
\end{tabular}

n/a-not applicable.

a The number of GAVI-eligible countries decreased from 75 to 72 in 2006.

b Four out of the 193 WHO member states for whom GNI data was not reported (Cook Islands, Nauru, Niue, and Tuvalu) were excluded from the income analysis. The GAVI

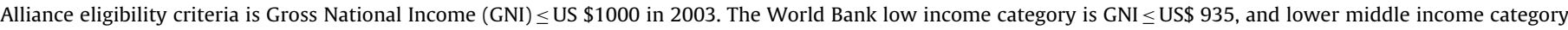

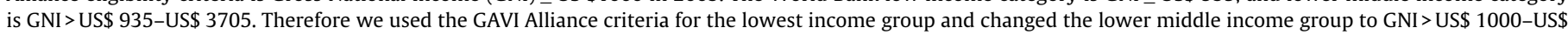
3705 .

that use the vaccine. By the end of 2009, the proportion of countries in each income category that use Hib vaccine ranged from a low of $65 \%$ among lower-middle income countries to $92 \%$ among high-income countries.

As shown in Table 1 and Fig. 3, nearly all WHO member states (34 [97\%] of 35) in the region of the Americas are using Hib vaccine, with only Haiti yet to introduce. Although Hib vaccine use in the European Region is high (47 [89\%] of 53), Eastern European countries have only started introducing recently. Among the eight GAVIeligible Eastern European countries, six have introduced since 2006 and the remainder have applied and been approved for GAVI support and plan to introduce in 2010. Use of Hib vaccine among countries in the Eastern Mediterranean region is also relatively high at $76 \%$ ( 16 of 21 ), although some non-GAVI eligible countries in this region with large birth cohorts have yet to introduce Hib vaccine. In the African region, 41 (89\%) of 46 countries have introduced Hib vaccine. Nigeria has applied for GAVI support and plans to introduce Hib vaccine in the next few years. The introduction of Hib vaccine in Nigeria would raise usage among GAVI-eligible countries in this region to $100 \%$. Hib vaccine use among countries in

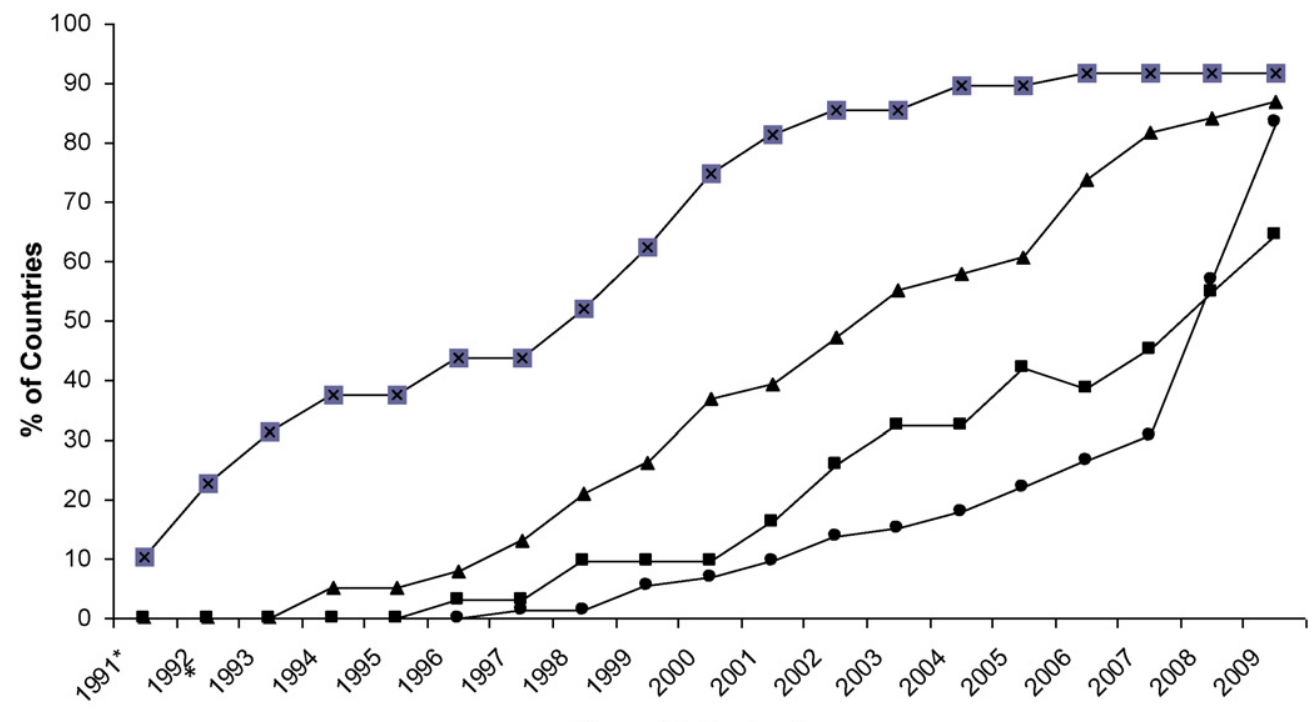

Year of Introduction

$\bullet$ GAVI-eligible ${ }^{\star \star} \rightarrow$ Lower middle income $\longrightarrow$ - Upper middle income $\quad-\mathbf{x}-$ High income

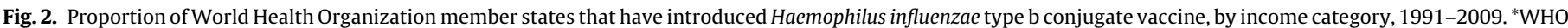

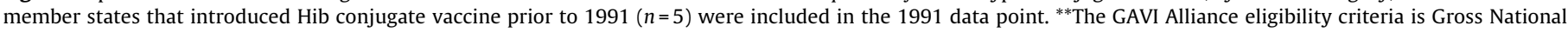

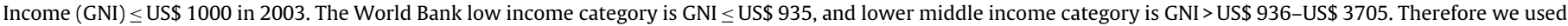
the GAVI Alliance criteria for the lowest income group and changed the lower middle income group to GNI > US\$ $1000-$ US\$ 3705 . 


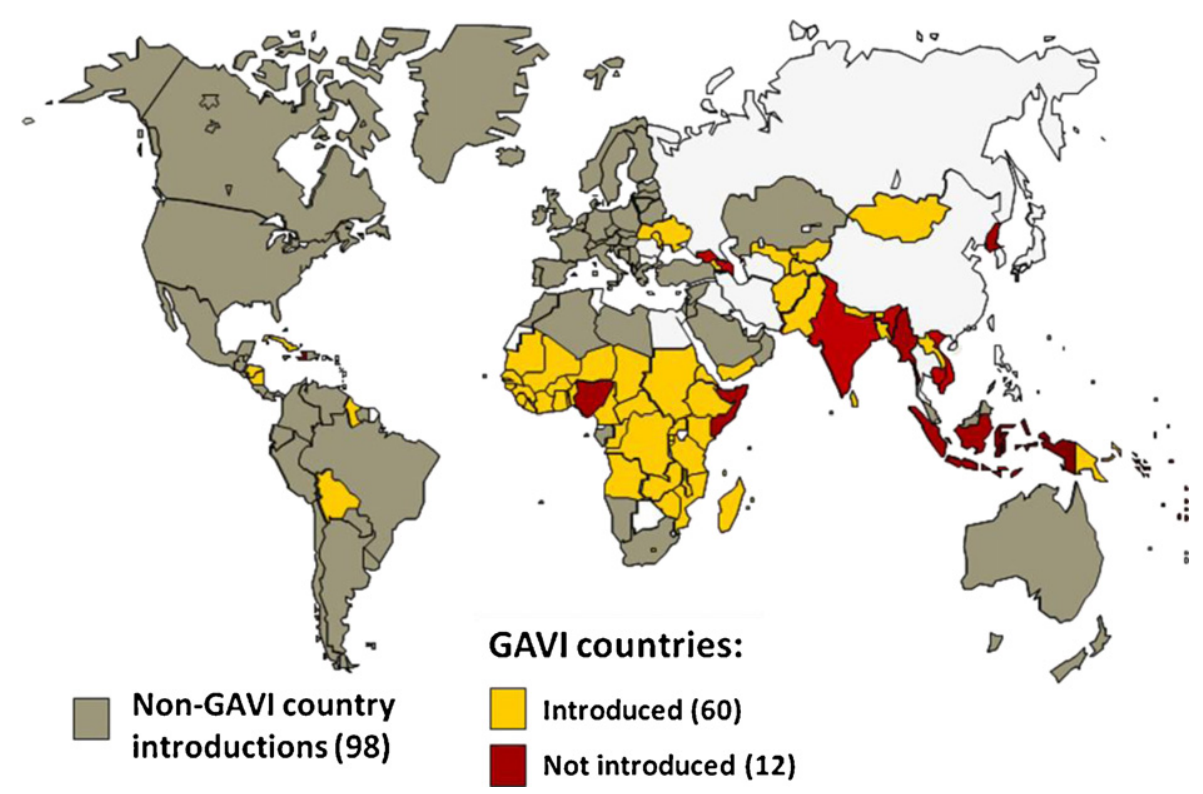

Fig. 3. Global status of Haemophilus influenzae type b conjugate vaccine use, 2009.

the Western Pacific region is 59\% (16 of 27), with Vietnam planning introduction in 2010 . Only four (36\%) of 11 countries in South East Asia have introduced Hib vaccine to date, and one country has been approved by GAVI for introduction. There has been significant progress in introduction in Asia over the past 3 years, and the countries that have not yet introduced are mainly developed countries, such as Japan, and those with large birth cohorts, such as India and China.

Of the 10 WHO member states with the largest birth cohorts globally, six (Pakistan, United States of America, Brazil, Ethiopia, Bangladesh, and Democratic Republic of the Congo) have introduced Hib vaccine. Additionally, Nigeria has applied for GAVI support for Hib vaccine introduction. Indonesia has included plans for Hib vaccine use in their national immunization comprehensive multi-year plan, which suggests that they plan to introduce the vaccine in the near future. India is approved to introduce the vaccine in 10 states, but the country is still evaluating introduction. Of the ten countries with the largest birth cohorts, only China has not yet decided to introduce Hib vaccine, although the Ministry of Health is currently considering introducing vaccine. Cumulatively, these ten countries make up 57\% of the global birth cohort. With a birth cohort of approximately 27 million, the universal introduction of Hib vaccine in India will raise global access to Hib vaccine by an additional $20 \%$. India is considering a phased introduction starting with 5-10 states in 2010 and full country introduction by 2012. Introduction in China, with a birth cohort of 17 million, would increase global access to Hib vaccine by another $13 \%$. Hib vaccine is believed to be widely available in China in the private market.

\section{Discussion}

Globally, use of Hib vaccine has increased tremendously since it became available in the late 1980s, with most of the progress in the last 3 years. Almost all high income countries had introduced by 2002. By the end of 2009, almost all GAVI-eligible, lowest income countries will have introduced Hib vaccine or been approved by GAVI to introduce Hib vaccine. Although it has taken nearly 20 years to introduce Hib vaccine into low income countries, the rate of vaccine introduction is generally faster than what would be expected based on the experience with hepatitis b vaccine, the last vaccine introduced into routine immunization systems worldwide [14]. However, it is important to recognize that although $82 \%$ of
WHO member states used Hib vaccine in their infant immunization schedules at the end of 2009 , only $45 \%$ of the world's children were fully vaccinated with Hib vaccine. This incongruity is largely due to the fact that a few countries with large birth cohorts (for example, India and China) have yet to introduce the vaccine.

A previous analysis found that a key factor for vaccine adoption before 2005 was country income level [15]. A more recent study found that GAVI support and cheaper vaccine price speeded vaccine introduction [16]. Our updated analysis highlights the slower progress in lower middle income countries. These countries may not have adequate financial resources or technical capacity to support vaccine introduction on their own and the financial support available to low income countries through the GAVI Alliance for new and underutilized vaccine introduction is not available to lower middle income countries, thus creating a widening gap in Hib vaccine uptake that did not exist 3 years ago. WHO and the Gates Foundation are currently working to assess the feasibility of various strategies that will help accelerate introduction of new vaccines in lower middle income countries.

Multiple factors contributed to the accelerated adoption of Hib vaccines in the last 4 years. First, in November 2006, WHO published a revised position statement recommending global use of Hib vaccine even in the absence of local disease burden data [17]. The statement provided clear guidance to countries that the burden of Hib disease has been well established and that vaccine efficacy and safety are well documented. Second, concerns about the ability of governments in GAVI-eligible countries to sustain the long-term cost of the vaccine after GAVI funding ends were partially alleviated. While GAVI originally financed the full cost of the vaccine for 5 years, countries were concerned about their ability to assume the full cost of the vaccine at the end of this period as the expected vaccine price declines were not seen. Additionally, many countries were uncertain that GAVI support would be available long-term [18]. GAVI's new co-financing model alleviated some of these concerns by ensuring long term support (until 2015) by providing a mechanism for the countries to gradually increase their contribution to the cost of the vaccine. Future price declines seem likely with a healthy vaccine market that includes over 30 existing $\mathrm{Hib}$ conjugate vaccines, including 12 that are WHO prequalified and at least 30 other Hib-containing products at various stages of development. Furthermore, uptake of the Hib vaccine may be attributed to the increasing popularity of the pentavalent vaccine, which con- 
tains Hib vaccine along with DTP and hepatitis $b$, as it has become an appealing option for countries because of its efficiency in protecting children against five different diseases; there are currently five pentavalent products prequalified by WHO.

The advent of the GAVI Alliance-supported Hib Initiative in 2005 served as a means to maximize the benefit from factors that were favorable for the introduction of Hib vaccine. The Hib Initiative was a consortium that comprised global partners from WHO, Switzerland; Johns Hopkins Bloomberg School of Public Health, USA; the London School of Hygiene and Tropical Medicine, United Kingdom; and the Centers for Disease Control and Prevention, USA. The goal of the Hib Initiative was to serve as a catalyst to mobilize activities focusing on $\mathrm{Hib}$ vaccine at the regional and national level and among global partners using a strategic approach of communication, coordination, research and surveillance. These activities included increasing awareness of Hib disease and Hib vaccine, through regional forums, country consultations, and support with the GAVI application process. The experience of the Hib Initiative may serve as a model to support introduction of other new and underutilized vaccines, such as pneumococcal conjugate and rotavirus vaccines [18].

Regional disparities in Hib vaccine use also persist, which is most notable in the low use of Hib vaccine in South East Asian countries. Despite studies showing that rates of Hib disease in Asia do not differ greatly from rates of Hib disease in other regions of the world $[19,20]$, adoption of the vaccine has been slow. However, use of Hib vaccine is increasing; two countries (Bangladesh and Nepal) introduced in early 2009 and two more are planning introductions by the end of 2009. This has been partly driven by publication of more recent studies from the region [21,22], a focused communication strategy, and funding of research projects by the GAVI Alliance and the Hib Initiative to support country decision-making.

This analysis has some limitations. Our data may have slightly overestimated the proportion of children with access to, and immunized with, Hib vaccine. Our access and coverage calculations were for each complete year and did not account for mid-year introduction. Birth cohort projections were used for 2008 and 2009, which may differ slightly from true birth cohorts. We used birth cohort data rather than live birth data or surviving infant data, which may have underestimated the proportion of children with access to or receiving Hib vaccine, since birth cohort data includes stillbirths and children who did not survive to a year of age. For countries that introduced Hib vaccine during 2009, we used 2008 DTP3 coverage data, the most recent data available, which may differ slightly from the true Hib3 coverage. Also, we relied on official WHO-UNICEF vaccine coverage estimates since they are consistently reported for all countries; however, these may overestimate actual vaccine coverage, particularly in countries reporting coverage following GAVI funding $[23,24]$. Our global estimates of access to Hib vaccine may underestimate the true number since vaccine is available on the private market in many countries, such as China, where it has not yet been added to the routine infant immunization schedule; however, data on private sector use of Hib vaccine is often proprietary and not widely available so we did not include it in our calculations. Similarly, we did not base our calculations on the number of vaccine doses delivered to countries. The annual number of countries we report as having introduced Hib vaccine differs slightly from WHO reports because we supplemented the WHO reports with additional information; specifically, we count Belarus and Sudan as having introduced by 2009 since Hib vaccine was introduced regionally into the immunization system in both countries and is widely available. For these reasons, our estimates may differ from other estimates reported by organizations such as WHO.

In keeping with Millennium Development Goal number four, further efforts are required to ensure sustained use of Hib vaccine in those countries already using it and continued advocacy for introduction in countries that have yet to make a decision. Increasing routine immunization coverage and strengthening the routine immunization system are key strategies in increasing Hib vaccine coverage. The disparity between access and coverage highlights the need for more efforts to ensure better implementation of vaccine programs and to overcome logistical challenges to deliver vaccines in the field. Strong disease surveillance programs to monitor disease trends and demonstrate the impact of Hib vaccine require ongoing commitment from countries and donors. WHO has developed manuals and tools that can be used to evaluate the impact of vaccine introduction on the vaccine program and on disease burden. For the few remaining GAVI-eligible countries that will not have yet introduced or made plans for introduction, stressed health systems, competing priorities, funding troubles within the GAVI Alliance, and difficult political climates have complicated and lengthened the decision making process. Despite great progress in the global introduction of Hib vaccine, special focus is needed for lower middle income countries that have not yet introduced.

\section{Acknowledgements}

We would like to thank the WHO new and underutilized vaccines focal persons from all WHO regions, Patrick Zuber, Gillian Mayers, Laure Dumolard and Hemanthi Dassanayake-Nicolas from WHO headquarters, and the Hib Initiative team, in particular Judy Heck and Marcie Richmond.

Conflict of interest statement: All authors report no conflicts.

\section{References}

[1] Watt J, Wolfson L, O’Brien K, Henkle E, Deloria-Knoll M, McCall N, et al. Burden of disease caused by Haemophilus influenzae type b in children younger than 5 years: global estimates. Lancet 2009;374(9693):903-11.

[2] Black RE, Cousens S, Johnson HL, Lawn JE, Rudan I, Bassani DG, et al. Global, regional, and national causes of child mortality in 2008: a systematic analysis. Lancet 2010;375(9730):1969-87.

[3] Jones G, Steketee R, Black R, Bhutta Z, Morris S. How many child deaths can we prevent this year? Lancet 2003;362(9377):65-71.

[4] UNICEF. Countdown to 2015 maternal, neonatal, and child survival. Tracking progress in maternal newborn and child survival. The 2008 report. New York: UNICEF; 2008.

[5] United Nations. United Nations millennium development goals. Available from: http://www.un.org/millenniumgoals/ [accessed November 13, 2008].

[6] Mulholland K, Hilton S, Adegbola R, Usen S, Oparaugo A, Omosigho C, et al. Randomised trial of Haemophilus influenzae type-b tetanus protein conjugate vaccine [corrected] for prevention of pneumonia and meningitis in Gambian infants. Lancet 1997;349(9060):1191-7.

[7] Adegbola RA, Secka O, Lahai G, Lloyd-Evans N, Njie A, Usen S, et al. Elimination of Haemophilus influenzae type $b$ (Hib) disease from The Gambia after the introduction of routine immunisation with a Hib conjugate vaccine: a prospective study. Lancet 2005;366(9480):144-50.

[8] Bisgard KM, Kao A, Leake J, Strebel PM, Perkins BA, Wharton M. Haemophilus influenzae invasive disease in the United States, 1994-1995: near disappearance of a vaccine-preventable childhood disease. Emerg Infect Dis 1998;4(2):229-37.

[9] Lewis RF, Kisakye A, Gessner BD, Duku C, Odipio JB, Iriso R, et al. Action for child survival: elimination of Haemophilus influenzae type b meningitis in Uganda. Bull World Health Organ 2008;86(4):292-301.

[10] World Health Organization. WHO vaccine preventable diseases monitoring system. Data, statistics and graphics, 1980-2008. Available at http://www. who.int/vaccines/globalsummary/immunization/countryprofileselect.cfm [accessed July 1, 2009].

[11] Vaccine Information Management System. Vaccine experience: Hib vaccine introductions in GAVI-eligible countries; 2009.

[12] United Nations. World population prospects: the 2008 revision population database. Available at http://esa.un.org/unpp/index.asp?panel=2 [accessed July 1,2009$]$

[13] World Bank. The World Bank country classification, data and statistics. Available at http://go.worldbank.org/K2CKM78CC0 [cited November 13, 2008].

[14] Levine O, Knoll M, Jones A, Walker D, Risko N, Gilani Z. Global status of Haemophilus influenzae type $\mathrm{b}$ and pneumococcal conjugate vaccines: evidence, policies, and introductions. Cur Opin Infect Dis 2010;23(3):23641.

[15] Rossi I, Zuber P, Dumolard L, Walker D, Watt J. Introduction of Hib vaccine into national immunization programmes: a descriptive analysis of global trends. Vaccine 2007;25(41):7075-80. 
[16] Shearer J, Stack M, Richmond M. Accelerating national decisions to adopt haemophilus influenzae type b vaccine: a global multivariate analysis. PLoS Med 2010;7(3):e1000249.

[17] World Health Organization. The WHO position paper on Haemophilus influenzae type b conjugate vaccines. Wkly Epidemiol Rec 2006;47(81):445-52.

[18] Hajjeh R, Privor-Dumm L, Edmond K, O'Loughlin R, Shetty S, Griffiths U, et al. Supporting new-vaccine introduction decisions: lessons learned from the Hib Initiative experience; Vaccine (2010), in press, [Epub ahead of print]

[19] Peltola H. Spectrum and burden of severe Haemophilus influenzae type b diseases in Asia. Bull World Health Organ 1999;77(11):878-87.

[20] Shetty S, Cohen A, Edmond K, Ojo L, Loo J, O'Loughlin R, et al. A systematic review and critical evaluation of invasive Haemophilus influenzae type B disease burden studies in Asia from the last decade: lessons learned for invasive bacterial disease surveillance. Pediatr Infect Dis J 2010;29(7):653-61.
[21] Baqui A, El Arifeen S, Saha S, Persson L, Zaman K, Gessner B, et al. Effectiveness of Haemophilus influenzae type B conjugate vaccine on prevention of pneumonia and meningitis in Bangladeshi children: a case-control study. Pediatr Infect Dis J 2007;26(7):565-71.

[22] Gessner B, Sutanto A, Linehan M, Djelantik I, Fletcher T, Gerudug I, et al. Incidences of vaccine-preventable Haemophilus influenzae type b pneumonia and meningitis in Indonesian children: hamlet-randomised vaccine-probe trial. Lancet 2005;365(9453):43-52.

[23] Lim S, Stein D, Charrow A, Murray C. Tracking progress towards universal childhood immunisation and the impact of global initiatives: a systematic analysis of three-dose diphtheria, tetanus, and pertussis immunisation coverage. Lancet 2003;372(9655):2031-46.

[24] Murray C, Shengelia B, Gupta N, Moussavi S, Tandon A, Thieren M. Validity of reported vaccination coverage in 45 countries. Lancet 2003;362(9389):1022-7. 